\title{
Relation of Serum Uric Acid Concentrations with Etiology and Severity in Patients with Cirrhosis of Liver
}

\author{
Rashedul Hasan ${ }^{*}$, Projesh Kumar Roy ${ }^{2}$, Md. Masudur Rahman Khan ${ }^{3}$ \\ ${ }^{1}$ Assistant professor, Department of Gastroenterology, Sheikh Russel National Gastroliver Institute \& Hospital, Mohakhali, Dhaka, Bangladesh \\ ${ }^{2}$ Professor \& Chairman, Department of Gastroenterology, BSMMU, Dhaka, Bangladesh \\ ${ }^{3}$ Associate Professor, Department of Gastroenterology, BSMMU, Dhaka, Bangladesh \\ Corresponding Author: Rashedul Hasan, Assistant Professor, Department of Gastroenterology, Sheikh Russel National Gastroliver \\ Institute \& Hospital, Mohakhali, Dhaka, Bangladesh
}

Received date: January 26, 2021; Accepted date: March 18, 2021; Published date: March 22,2021

Citation: Hasan R., Projesh K. Roy, Masudur R. Khan (2021) Relation Of Serum Uric Acid Concentrations With Etiology And Severity In Patients With Cirrhosis Of Liver J. Gastroenterology Pancreatology and Hepatobilary Disorders 5(2) DOI:10.31579/2641-5194/019

Copyright: (c) 2021, Rashedul Hasan,This is an open access article distributed under the Creative Commons Attribution License, which permits unrestricted use, distribution, and reproduction in any medium, provided the original work is properly cited.

\begin{abstract}
Background: Hyperuricemia is now an established factor to cause oxidative stress, insulin resistance and systemic inflammation. So it is likely that hyperuricemia might be involved in hepatic necro-inflammation and destruction which are the common underlying pathophysiology of cirrhosis. On the other hand, as uric acid is the end product of cellular degradation, increased hepatocyte destruction due to any etiology increases the level of serum uric acid which might further aggravate hepatic necro-inflammation, cirrhosis \& complications.
\end{abstract}

Objectives: To assess serum uric acid concentrations in patients of cirrhosis of liver and its relation with cirrhosis of different etiology, disease severity and liver enzymes.

Materials and Methods: This cross sectional observational study was carried out in the Department of Gastroenterology, BSMMU, Bangladesh during the period of September 2015 to October 2016. A total of 220 diagnosed cases of cirrhosis of liver due to any cause from inpatient \& outpatient Department of Gastroenterology of Banghabandhu Sheikh Mujib Medical University were enrolled as the study population. Serum uric acid level was measured in each patient and its relationship with different etiology of cirrhosis, severity of cirrhosis and liver enzymes were assessed.

Results: The mean age was found to be $47.8 \pm 14.6$ years and male: female ratio was 1.9:1. Majority patients $(52.3 \%)$ belonged to CTP Class C. The mean $( \pm \mathrm{SD})$ value of serum uric acid was $6.19( \pm 3.25 \mathrm{mg} / \mathrm{dl})$ and hyperuricemia $(>7 \mathrm{mg} / \mathrm{dl})$ was detected in $27.73 \%$ patients. Among all etiologies of CLD, the higher mean ( \pm SD) level of serum uric acid was found in NAFLD $(19.54 \pm 2.20 \mathrm{mg} / \mathrm{dl})$. There was positive correlation of serum uric acid with liver enzymes.

Conclusion: Mean serum uric acid level increased gradually as the cirrhotic patients progressed to higher CTP classes $\&$ there was positive correlation of serum uric acid with liver enzymes. It requires further large scale multicenter studies with increased sample size \& prolong follow-up to establish serum uric acid as a risk factor of CLD.

Keywords: cirrhosis, factor Of CLD, NAFLD, serum uric acid, liver enzymes.

\section{Introduction}

Cirrhosis is a diffuse process characterized by fibrosis and the conversion of normal liver architecture into structurally abnormal regenerative nodule [1]. Cirrhosis may be compensated; or decompensated when complicated by one or more of the following features: jaundice, ascites, hepatic encephalopathy and raised prothrombin time. Cirrhosis is irreversible. Unless the underlying cause of cirrhosis is removed and the person takes measures to treat the condition, the liver will continue to incur damage, eventually leading to liver failure, ammonia toxicity, gastrointestinal hemorrhage, kidney failure, hepatic coma, and death [2]. Common complications of cirrhosis include GI bleeding, ascites, encephalopathy, hepatorenal syndrome, SBP and others. Continued inflammatory process and hepatocyte destruction due to the activity of underlying etiology (i.e. chronic hepatitis B \&/or chronic hepatitis C virus, alcohol etc) is responsible for advancement of the cirrhotic process and leads decompensation and occurrence of complications. In humans and higher primates, uric acid (UA) is the final oxidation product of purine metabolism and is excreted in urine. Hyperuricemia is defined as a serum urate concentration greater than $7 \mathrm{mg} / \mathrm{dl}(416 \mu \mathrm{mol} / \mathrm{L})$, the approximate level at which urate is supersaturated in plasma [3]. Hyperuricemia has long been recognized as a cause of gouty arthritis and kidney stones [4]. In recent years, hyperuricemia has also been implicated in the development of many other diseases. Obvious correlation has been found in hypertension, kidney disease, metabolic syndrome, and cardiovascular disease $[5,6]$. The underlying mechanisms include induction of endothelial dysfunction, insulin resistance, oxidative stress, 
and systemic inflammation by hyperuricemia [5, 6]. Hyperuricemia is now an established factor to cause oxidative stress, insulin resistance and systemic inflammation. So it is likely that hyperuricemia might be involved in hepatic necro-inflammation [4]. In fact, different studies across the world have found correlation between hyperuricemia and cirrhosis. Benerji et al. [4], conducted comparative study of ALT, AST, GGT \& uric acid levels in liver diseases and found association between serum uric acid levels and the incidence of cirrhosis-related hospitalization or the presence of elevated serum ALT or GGT. These associations were largely independent of other known liver disease risk factors. According to their study, the elevated serum uric acid level might be a risk factor for the incidence of chronic liver disease. In an observational study by Paul et al. [7], found significantly high serum uric acid level in certain cases of CLD i.e. chronic viral hepatitis and autoimmune hepatitis. Uric acid levels were higher in higher CTP score and it was also linked with mortality. Serum uric acid level showed correlation with parameters of CLD i.e. SGOT and INR. Lee et al. [8], showed in a study on Korean adults with non-alcoholic fatty liver disease that Serum uric acid is independently associated with the presence of NAFLD, and uric acid may be a useful additional measure in assessing the risk of NAFLD in the clinical setting. These associations remained even after adjustment for other surrogate markers of NAFLD, such as AST, ALT, GGT and CRP. Petta et al. [9], carried out a study on a cohort of patients with histological diagnosis of NAFLD and showed an independent link between hyperuricemia and the severity of liver damage. In particular, they found that hyperuricemia was independently associated with the severity of steatosis and lobular inflammation. Petta et al. [9], carried out another study on a cohort of mostly overweight or obese patients with histological diagnosis of chronic hepatitis $\mathrm{C}$ and found that hyperuricaemia was independently associated with steatosis, its severity and also a factor involved in the progression of liver disease. A crucial question was raised in different studies as to whether hyperuricaemia plays any role in directly causing hepatic necro-inflammation and cirrhosis or whether it is a marker for an adverse metabolic profile that leads to NAFLD/NASH or promotes progression of viral or alcoholic hepatitis [10].

\section{Aims and objectives}

\section{General Objective:}

To assess uric acid concentrations in patients of cirrhosis of liver and its relation with etiology \& severity.

\section{Specific Objectives:}

$\square$ To see the uric acid concentration in different classes of cirrhosis on the basis of Child Turcotte Pugh score.

To determine the association of uric acid concentration with cirrhosis of different etiology.

\section{Methods and Materials}

This cross sectional observational study was carried out in the Department of Gastroenterology, BSMMU, and Bangladesh during the period of September 2015 to October 2016. A total of 220 diagnosed cases of cirrhosis of liver due to any cause from inpatient \& outpatient Department of Gastroenterology of Banghabandhu Sheikh Mujib Medical University were enrolled as the study population. Serum uric acid level was measured in each patient and its relationship with different etiology of cirrhosis, severity of cirrhosis and liver enzymes were assessed. Patients were excluded below the age 18, known malignancy, on chemotherapy, on drugs that may impair uric acid level such as allopurinol/ feboxostat/diuretics, known infection, recent surgery/trauma from the study. History, clinical examination \& laboratory investigations were noted in a semi structured data sheet. ALT, AST, endoscopy upper git, USG was done in all patients. The results were noted on the data sheet. Etiology of cirrhosis was ascertained by appropriate investigations, if not known previously, i, e $\mathrm{HbsAg}$, anti $\mathrm{Hbc}$ total, Anti $\mathrm{HCV}$, serum caeruloplasmin Urinary copper was done and noted in data sheet. Serum uric acid level was measured for single time for all patients, and send to biochemistry department, the result was noted in the data sheet. CTP score was done for each patient.

\section{Inclusion criteria:}

- Patients who are diagnosed to have cirrhosis of liver

- Patients more than 18 years of age

\section{Exclusion criteria:}

- Malignancy

- Patients on Chemotherapy

- Patients taking drugs such as diuretics, salicylates, pyrazinamide, ethambutol, nicotinic acid, ciclosporin, 2ethylamino-1,3,4-thiadiazole, allopurinol, febuxostat and cytotoxic agents

- Recent surgery

Data Collection: Prior to data collection both verbal and written consent was taken from the respondents. Data were collected using a preformed data collection sheet (questionnaire). Base line information was collected from the patient after exploration of different complaints. All information regarding clinical features was recorded in a data collection sheet. All the investigations weredone in the Bangabandhu Sheikh Mujib Medical University, Dhaka, Bangladesh.

Statistical analysis: All the data were checked and edited after collection. Then the data were entered into computer and statistical analysis of the results being obtained by using windows based computer software devised with Statistical Packages for Social Sciences (SPSS-22) (SPSS Inc, Chicago, IL, USA). The results were presented in tables and figures. Statistical significance was set at $\mathrm{p}<0.05$ and confidence interval set at $95 \%$ level. Continuous variables were expressed as mean with standard deviation and categorical variables as count with percentage. Categorical data were assessed by Chi-square test. Association between uric acid and different etiology and CTP class were assessed by ANOVA test. The correlation between variables were be evaluated by means of Pearson's correlation test.

Operational definitions: Cirrhosis of liver: Cirrhosis, a final pathway for a wide variety of chronic liver diseases, is a pathologic entity defined as diffuse hepatic fibrosis with the replacement of the normal liver architecture by nodules. Cirrhosis is strictly speaking a histological diagnosis, a combination of clinical, laboratory, and imaging features can help confirm a diagnosis of cirrhosis [11]. Physical findings suggestive of cirrhosis of liver are, palmer erythema, gynecomastia, spider telengiectasias or angioma, caput medusae, parotid enlargement, enlarge left lobe of liver with splenomegaly suggests cirrhosis of liver especially in setting of thrombocytopenia and impaired hepatic function ( e.g., hypoalbuminemia, prolongation of prothrombin time). Clinical history of chronic liver disease with gastro-oesophageal varices, ascites, or hepatic encephalopathy is likely to have cirrhosis, and liver biopsy is not essential for confirming cirrhosis. Fibroelastography or Magnetic resonance elastography can help confirm a diagnosis of cirrhosis. Liver biopsy has long been the gold standard for diagnosing cirrhosis but may be associated with costs and procedure related risks, albeit infrequently. The ideal combination of clinical findings and routine laboratory tests to determine whether a patient has cirrhosis without need for liver biopsy has been addressed in systematic fashion [12]. 


\begin{tabular}{|l|c|c|c|}
\hline & \multicolumn{3}{|c|}{ Points* } \\
\hline Encephalopathy & 1 & 2 & 3 \\
\hline Ascites & None & $\begin{array}{c}\text { Grade } 1-2 \\
\text { (or precipitant-induced) }\end{array}$ & $\begin{array}{c}\text { Grade } 3-4 \\
\text { (or chronic) }\end{array}$ \\
\hline Bilirubin (mg/dL) & None & $\begin{array}{c}\text { Mild/Moderate } \\
\text { (diuretic-responsive) }\end{array}$ & $\begin{array}{c}\text { Severe } \\
\text { (diuretic-refractory) }\end{array}$ \\
\hline Albumin (g/dL) & $<2$ & $2-3$ & $>3$ \\
\hline $\begin{array}{l}\text { PT (sec prolonged) } \\
\text { or INR }\end{array}$ & $>3.5$ & $2.8-3.5$ & $>2.8$ \\
\hline
\end{tabular}

CTP score is obtained by adding the score for each parameter.

CTP class: $A=5-6$ points, $B=7-9$ points, $C=10-15$ points.

\section{Result and Observation}

This cross sectional study was carried out in the Department of Gastroenterology, BSMMU, Dhaka, Bangladesh during the period of September 2015 to October 2016 involving 220 diagnosed patients of cirrhosis of liver due to any etiology to investigate serum uric acid level in cirrhosis of liver patients and its relation with cirrhosis of different ation of the severity of cirrhosis

etiology, disease severity. Among 220 cirrhotic patients, maximum 63 (28.6\%) patients were in the age group of 51-60 years followed by 43 $(19.5 \%), 41(18.6 \%), 37(16.8 \%)$ and $36(16.4 \%)$ patients were in the age group of 41-50 years, 31-40 years, $>60$ years and $\leq 30$ years respectively. Mean age was $47.8 \pm 14.6$ years. Male $(65.5 \%)$ were predominant than female $(34.5 \%)$. Female male ratio was 1:1.9 (Table 1$)$.

\begin{tabular}{|l|c|c|}
\hline Parameters & Frequency (n) & Percentage (\%) \\
\hline Age & & 16.4 \\
\hline$\leq 30$ & 36 & 18.6 \\
\hline $31-40$ & 41 & 19.5 \\
\hline $41-50$ & 43 & 28.6 \\
\hline $51-60$ & 63 & 16.8 \\
\hline$>60$ & 37 & \\
\hline & Mean \pm SD: $47.8 \pm 14.6$ & \\
\hline Gender & & 65.5 \\
\hline Male & 144 & 34.5 \\
\hline Female & 76 & $\mathbf{1 0 0 . 0}$ \\
\hline Total & $\mathbf{2 2 0}$ & \\
\hline
\end{tabular}

Table 1. Distribution of patients according to age and gender.

Among 220 patients, 159 (72.27\%) patients had normal level of serum uric acid $(\leq 7 \mathrm{mg} / \mathrm{dl})$ and the rest $61(27.73 \%)$ patients had hyperuricemia $(>7$ $\mathrm{mg} / \mathrm{dl}$ ). (Figure 1).

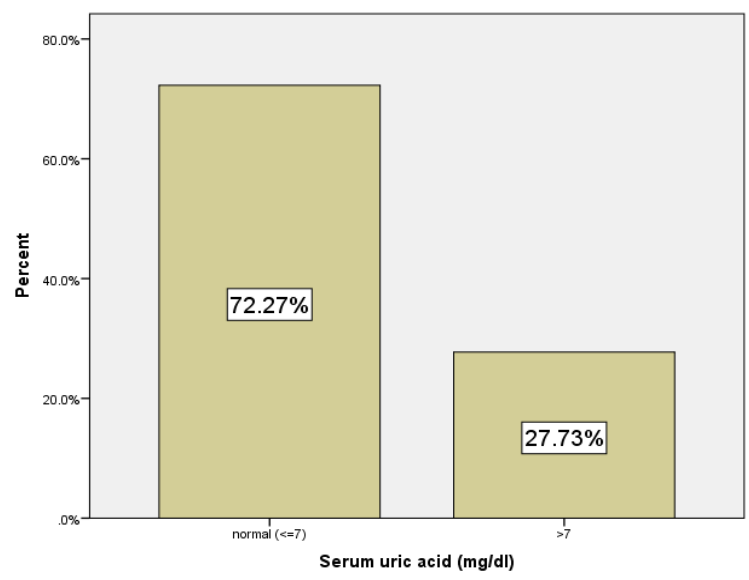

Figure-1: Bar Diagram on serum uric acid distribution in 220 cirrhotic patients. 
The mean serum uric acid level increased gradually as the patients progressed to higher CTP classes. Mean serum uric acid level was highest in CTP class C patients followed by CTP class B and class A. (Table 2).

\begin{tabular}{|l|c|c|c|c|}
\hline CTP class & $\mathrm{n}(\%)$ & $\begin{array}{c}\text { Hyperuricemia } \\
\mathrm{n}(\%)\end{array}$ & Mean SD & Range (Min-Max) \\
\hline $\mathrm{A}$ & $48(21.8)$ & $2(3.3)$ & $4.30 \pm 1.64$ & $2.30-9.80$ \\
\hline $\mathrm{B}$ & $57(25.9)$ & $13(21.3)$ & $5.60 \pm 1.83$ & $3.10-9.80$ \\
\hline C & $115(52.3)$ & $46(75.4)$ & $6.98 \pm 3.80$ & $1.70-21.00$ \\
\hline Total & $220(111.0)$ & $61(100.0)$ & $6.19 \pm 3.25$ & $1.70-21.00$ \\
\hline
\end{tabular}

Statistical analysis

\begin{tabular}{|l|c|}
\hline Groups & p value \\
\hline A vs B vs C & $<0.001$ \\
\hline A vs B & 0.210 \\
\hline A vs C & $<0.001$ \\
\hline B vs C & 0.027 \\
\hline
\end{tabular}

One way ANOVA followed by Bonferroni test was performed to compare between groups.

Table 2. Relation of Uric acid with severity of liver cirrhosis according to CTP class.

Bar diagram of relation of Serum Uric Acid with severity of liver cirrhosis according to CTP class. (Figure-2)

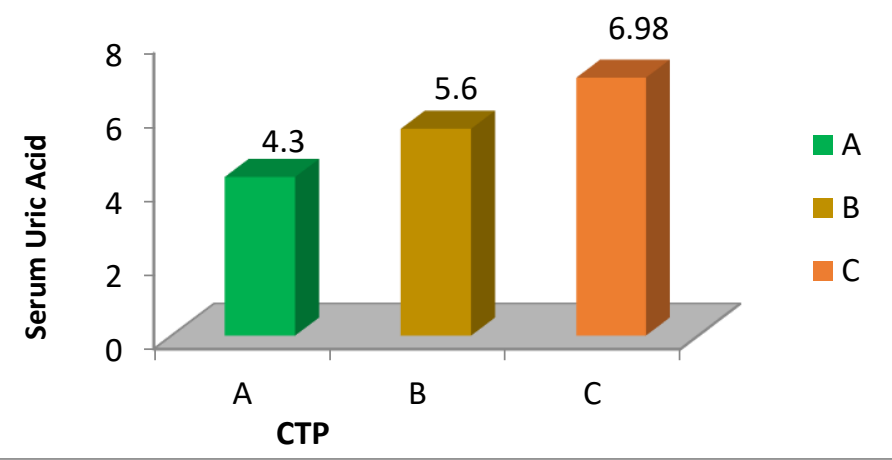

Figure-2: Bar diagram of relation of Serum Uric Acid with severity of liver cirrhosis according to CTP class.

Among the 220 patients of cirrhosis of different etiology, the mean serum uric acid level was found maximum among NAFLD patients $(19.54 \pm 2.20$ $\mathrm{mg} / \mathrm{dl}$ ) followed by cirrhosis of liver due to $\mathrm{CHC}$, Wilson disease \& $\mathrm{CHB}$ where the mean of serum uric acid level was $6.74( \pm 2.75) \mathrm{mg} / \mathrm{dl}, 5.91$ $( \pm 2.76) \mathrm{mg} / \mathrm{dl} \& 5.61( \pm 2.19) \mathrm{mg} / \mathrm{dl}$ respectively. (Table $3 \& 4$ )

\begin{tabular}{|c|c|c|c|c|}
\hline Diagnosis & $\mathrm{n}(\%)$ & $\begin{array}{c}\text { Hyperuricemia } \\
\text { n }(\%)\end{array}$ & Mean SD & Range (Min-Max) \\
\hline $\mathrm{CHB}$ & $119(54.1)$ & $28(23.5)$ & $5.61 \pm 2.19$ & $1.70-11.20$ \\
\hline $\mathrm{CHC}$ & $49(22.3)$ & $16(32.7)$ & $6.74 \pm 2.75$ & $3.30-14.10$ \\
\hline Wilson's disease & $18(8.2)$ & $5(27.8)$ & $5.91 \pm 2.76$ & $2.30-10.00$ \\
\hline NAFLD & $5(2.3)$ & $5(100.0)$ & $19.54 \pm 2.20$ & $15.70-21.00$ \\
\hline Non $\mathrm{B} /$ non $\mathrm{C}$ & $29(13.2)$ & $7(24.1)$ & $4.96 \pm 2.20$ & $2.30-8.00$ \\
\hline Total & $220(100.0)$ & $61(27.7)$ & $6.19 \pm 3.25$ & $1.70-21.00$ \\
\hline
\end{tabular}

Table 3. Serum uric acid concentrations in different etiology of liver cirrhosis patients.

\begin{tabular}{|l|c|c|c|c|}
\hline Diagnosis & $\mathrm{n}(\%)$ & Mean SD & Range (Min-Max) & P value \\
\hline Non NAFLD & $215(97.7)$ & $5.83 \pm 2.42$ & $1.70-14.10$ & 0.001 \\
\hline NAFLD & $5(2.3)$ & $19.54 \pm 2.20$ & $15.70-21.00$ & \\
\hline Total & $220(100.0)$ & $6.19 \pm 3.25$ & $1.70-21.00$ & \\
\hline
\end{tabular}

Table 4. Serum uric acid concentrations in NAFLD and other causes of liver cirrhosis patients.

Among 61 patients of hyperuricemia, maximum $46(75.4 \%)$ patients were in CTP class C followed by $13(21.3 \%)$ and $2(3.3 \%)$ cases in CTP class $\mathrm{B}$ and A respectively. The association of serum uric acid (normal and \begin{tabular}{|l|c|}
\hline CTP class & Hyperuricemia \\
$\mathrm{n}(\%)$
\end{tabular} hyperuricemia) with severity of liver cirrhosis according to CTP class was statistically significant. (Table 5)

Normal
$\mathrm{n}(\%)$

$\mathrm{n}(\%)$
$P$ value 


\begin{tabular}{|l|c|c|c|}
\hline A & $2(3.3)$ & $46(28.9)$ & $<0.001$ \\
\hline B & $13(21.3)$ & $44(27.7)$ & 0.334 \\
\hline C & $46(75.4)$ & $69(43.4)$ & $<0.001$ \\
\hline Total & $\mathbf{6 1 ( 1 0 0 . 0 )}$ & $\mathbf{1 5 9 ( 1 0 0 . 0 )}$ & \\
\hline Chi & & \\
\hline
\end{tabular}

Chi-square test was done to measure the level of significance

Table 5. Association of serum uric acid (normal and hyperuricemia) with severity of liver cirrhosis according to CTP class.

\section{Discussion}

In this study a total of 220 cirrhotic patients of any etiology were investigated for serum uric acid level and its relationship with different etiology of cirrhosis, severity of cirrhosis and liver enzymes were assessed. According to patient's age, mean age was $47.8 \pm 14.6$ years with an age range of $18-75$ years. Maximum $63(28.6 \%)$ patients were in the age group of 51-60 years followed by 43 (19.5\%), 41 (18.6\%), 37 (16.8\%) and $36(16.4 \%)$ were in the age group of $41-50$ years, $31-40$ years, $>60$ years and $\leq 30$ years respectively. A recent survey Shih et al. [13], determined the association between nonalcoholic fatty liver disease (NAFLD) and serum uric acid (SUA) levels. The mean age of his study subjects was 40.8 years. Another prospective study by Xu et al. [14], on NAFLD \& serum uric acid involving 7412 participants found the mean age to be 44.4 years. These were nearer to our study in both the instances. Considering gender distribution, male $(65.5 \%)$ was predominant than female $(34.5 \%)$ and the ratio was $1.9: 1$. In a recent study revealing the association of serum uric acid and liver diseases by Shih et al. [13], reported $47.6 \%$ study population were male. Another study by Paul et al. [7], found that $31 \%$ were female among their study population while carrying out study on serum uric acid level in chronic liver disease. These were also consistent with this study result. In our study, the focus of our laboratory variable was serum uric acid. The mean serum uric acid was $6.19( \pm 3.25) \mathrm{mg} / \mathrm{dl}$. The range was found between 1.70 to $21.00 \mathrm{mg} / \mathrm{dl}$. Among 220 patients, 159 patients had normal level of uric acid $(\leq 7$ $\mathrm{mg} / \mathrm{dl}$ ) which represents about $72.27 \%$ and the rest 61 of patients i.e. $27.73 \%$ had hyperuricemia $(>7 \mathrm{mg} / \mathrm{dl}$ ). A national survey on association between serum uric acid and nonalcoholic fatty liver disease in the US population reported that overall $16.4 \%$ had hyperuricemia [13]. A study in India by Benerji, et al. [4], found the mean level of serum uric acid was $10.6( \pm 2.0) \mathrm{mg} / \mathrm{dl}$ among 55 cirrhotic patients. Antony et al. [15], measured the serum uric acid to be $5.63( \pm 0.08) \mathrm{mg} / \mathrm{dl}$ in liver disease patients. The most common cause of cirrhosis of liver in this study was chronic HBV infection followed by chronic HCV, Wilson disease, Non $\mathrm{B} /$ Non $\mathrm{C}$ and NAFLD. Among the 220 patients of liver cirrhosis, the frequency of $\mathrm{CHB}$ was $54.1 \%$ followed by chronic $\mathrm{HCV}$ in $22.3 \%$, Wilson disease in $8.2 \%$, NAFLD in $2.3 \%$ and Non B/ non C in $13.2 \%$ patients. Alcoholic was found to be the commonest $(36.5 \% ; \mathrm{n}=19)$ commonest cause followed by NAFLD (28.8\%), Wilsons disease, autoimmune hepatitis and cryptogenic cirrhosis in a study by Paul et al. [7], titled as 'Study of serum uric acid in chronic liver disease and its relation with other parameters. Considering the relation of uric acid with etiology of liver cirrhosis, mean serum uric acid was found maximum in NAFLD patients followed by Cirrhosis liver disease due to CHC, Wilson disease and CHB patients. The mean (SD) level of serum uric acid in NAFLD was found $19.54( \pm 2.20) \mathrm{mg} / \mathrm{dl}$. Shih et al. [13], found a significant association between serum uric acid levels and NAFLD among the US population. Moreover, Afzali et al. [10], have showed that serum uric acid levels were associated with the progression of chronic liver diseases such as NAFLD and NASH; and hyperuricemia was found independently associated with the severity of liver damage among NAFLD patients. In a cohort study in China which followed 6890 men and women without NAFLD for 3 years and found a higher incidence of NAFLD in those with higher baseline serum uric acid levels [14]. Another cross-sectional study showed that NAFLD patients had higher serum uric acid levels than healthy controls and that the prevalence of NAFLD was increased at higher serum uric acid levels, suggesting significant association between serum uric acid and NAFLD [16]. Severity of Cirrhosis of liver was assessed by CTP score in this study and among 220 cirrhotic patients $21.8 \%$ belonged to CTP Class A, 25.9\% to CTP Class $\mathrm{B}$ and the majority i.e. 52.3\% belonged to CTP Class C. Paul et al. [7], reported in a similar study that $55.76 \%$ of his patients belonged to CTP Class B \& $69.2 \%$ fell into combined CTP Class B and C. Regarding the relationship of uric acid with severity of liver cirrhosis according to CTP class, mean serum uric acid level increased gradually as the patients progressed to higher CTP class. Mean serum uric acid level (mg/dl) was maximum in CTP class $\mathrm{C}$ patients $(6.98 \pm 3.80)$ followed by class $\mathrm{B}(5.60$ $\pm 1.83)$ and class $\mathrm{A}(4.30 \pm 1.64)$. This increase of serum uric acid was statistically significant. Paul et al. [7], reported serum uric acid levels were significantly higher in CTP classes B and C as compared to CTP class A $(p<0.0001)$ \& serum uric acid levels were higher with higher CTP scores $(r=0.44)$. These findings were consistent with our findings. On observing the liver enzymes level, the mean level of ALT and AST was found to be $48.61 \pm 45.48 \mathrm{U} / \mathrm{L}$ and $61.33 \pm 53.35 \mathrm{U} / \mathrm{L}$ respectively. In this study, the correlation of uric acid with AST \& ALT, there was significant positive correlation of serum uric acid with AST ( $r$ value $=0.236$; $\mathrm{p}=0.003$ ). Uric acid had positive correlation with ALT also but the relationship was not statistically significant. Shih et al. [13], also found that patients with hyperuricemia were more likely to have elevated liver enzymes. Findings of liver enzymes by Benerji, et al [4], in liver cirrhosis patients correlate with our study findings. They found the ALT level as $67 \pm 31.4 \mathrm{U} / \mathrm{L}$ and AST as $65 \pm 27.5 \mathrm{U} / \mathrm{L}$. Paul et al. [7], also found that serum uric acid levels showed significant correlation with AST $(r=0.464)$ in patients with cirrhosis which is consistent with our study findings.

\section{Conclusion}

In conclusion, $27.73 \%$ among 220 cirrhotic patients had hyperuricemia. Among all etiological causes of liver cirrhosis, serum uric acid was higher among NAFLD patients. Significant positive correlation of serum uric acid level has been found with serum AST. Mean serum uric acid level increased gradually as the patients progressed to higher CTP classes. Based on this observation, serum uric acid could be considered as a marker of severity of CLD but it requires further large scale multicenter studies with increased sample size \& prolong follow-up to establish it as a surrogate marker.

\section{Limitation and Recommendation}

- The study was done in limited time of span; cases were collected from only one center hence may not represent the whole population of the country.

- $\quad$ The sample size was small.

- There was no control group in this study.

- Further multi centered prospective cohort study with large sample size with follow-up for prolong period is recommended.

\section{References}

1. Hawkey, C.J., Bosch J., Joel, E., Garcia, G., and Francis, K.L. ( $\left(2^{\text {nd }}\right.$ edition) (1992). 'Ascites', 'Textbook of clinical gastroenterology and hepatology', Willey -Blackwell, U.K, pp. 103-06. 
2. Dooley, J.S., Lok, A., Burroughs, A.K. and Heathcote, J. eds. 2011. 'Sherlock's diseases of the liver and biliary system. $12^{\text {th }} \mathrm{edn}$. USA. Wiley-Blackwell.

3. Loeb, J.N., 1972. 'The influence of temperature on the solubility of monosodium urate'. Arthritis \& Rheumatism, vol. 15(2), pp.189-92.

4. Benerji, V., Babu, M.F., Das, R.K., Saha, A., 2013. 'Comparative Study of ALT, AST, GGT \& Uric Acid Levels in Liver Diseases', Journal of Dental and Medical Sciences, vol. 7(5), pp. 72-5

5. Feig, D.I., Kang, D.H. and Johnson, R.J., 2008. 'Uric acid and cardiovascular risk', New England Journal of Medicine, vol. 359(17), pp.1811-21.

6. Edwards, N.L., 2009. 'The role of hyperuricemia in vascular disorders', Current opinion in rheumatology, vol. 21(2), pp.1327.

7. Paul, R., Chakravarti, H.N., Mandal, S.K., Chatterjee, S., Choudhury, P.S., 2013. 'Study of serum uric acid in chronic liver disease and its relation with other parameters', International Research Journal of Pharmacy, vol. 4 (7), pp. 162-5.

8. Lee, Y.J., Lee, H.R., Lee, J.H., Shin, Y.H. and Shim, J.Y., 2010. 'Association between serum uric acid and non-alcoholic fatty liver disease in Korean adults', 'Clinical chemistry and laboratory medicine, vol. 48(2), pp.175-80.

9. Petta, S., Camma, C., Cabibi, D., Di Marco, V. and Craxi, A., 2011. 'Hyperuricemia is associated with histological liver damage in patients with non-alcoholic fatty liver disease', Alimentary pharmacology \& therapeutics, vol. 34(7), pp.757-66.
10. Afzali, A., Weiss, N.S., Boyko, E.J., Ioannou, G.N., 2010. 'Association between serum uric acid level and chronic liver disease in the United States', Hepatol, vol. 52, pp.578-89.

11. Ramachandran, A. and Balasubramanian, K.A., (2001) 'Intestinal dysfunction in liver cirrhosis: Its role in spontaneous bacterial peritonitis', Journal of gastroenterology and hepatology, vo. 16(6), pp.607-12.

12. Udell, J., Wang, C., Tinmouth, J., FitzGerald, J., Ayas, N., Simel, D., Schulzer, M., Mak, E. and Yoshida, E. (2012). Does This Patient With Liver Disease Have Cirrhosis?, Journal of American Medical Association, vol. 307(8).

13. Ming-Hsiunga, S., Lazo, M., Liu, S., Bonekamp, S., Hernaez, R., Jeanne, M., 2015, 'Association between serum uric acid and nonalcoholic fatty liver disease in the US population', Journal of the Formosan Medical Association; vol. 114: pp. 314-20

14. Xu, C., Yu, C., Xu, L., Miao, M. and Li, Y., 2010. 'High serum uric acid increases the risk for nonalcoholic Fatty liver disease: a prospective observational study', PloS one, vol. 5(7), pp. 11578.

15. Antony, T.P., David, I., Thomas, A., George K.S., Ambookan, R., Menon, S., 2012. 'Correlation of Serum Uric Acid levels to the prevalence and severity of non-alcoholic fatty liver disease: A cross sectional study in south Indian males', IMAKMJ, vol. 40, pp. 188-93.

16. Li, Y., Xu, C., Yu, C., Xu, L., Min, M., 2009. 'Association of serum uric acid level with non-alcoholic fatty liver disease: a cross-sectional study', J Hepatol, vol. 50, pp. 1029-34.
This work is licensed under Creative Commons Attribution 4.0 License

\section{To Submit Your Article Click Here: Submit Manuscript}

DOI: $10.31579 / 2641-5194 / 019$

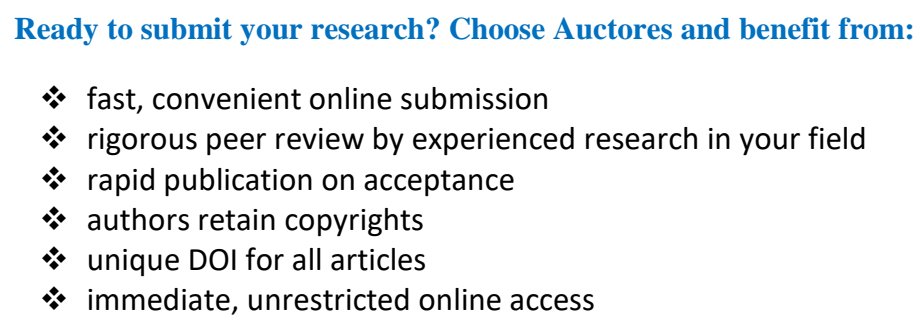

At Auctores, research is always in progress.

Learn more www.auctoresonline.org/journals/gastroenterologypancreatology-and-hepatobilary-disorders 